\title{
The Worst Case for Real Options
}

\author{
M. Trojanowska $\cdot$ P.M. Kort
}

Published online: 15 April 2010

(C) The Author(s) 2010. This article is published with open access at Springerlink.com

\begin{abstract}
The problem of the timing of an investment decision under partial information is analyzed in a framework where the firm is ambiguity averse. The analysis yields the description of a robust decision rule for an investment in a finite life project in presence of a stochastic instantaneous return. It is demonstrated that ambiguity aversion may accelerate investment in the short run. Ex post validation of the determined investment policy treats the impact of ambiguity aversion on the proper way of discounting of the profit flow resulting from the project and the fair price of risk associated with ambiguity aversion.
\end{abstract}

Keywords Real options - Ambiguity aversion - Endogenous discount factor · Market price of risk $\cdot$ Ex post analysis

\section{Introduction}

The estimation of a project's growth rate is a crucial problem for determining the timing of an investment decision. This paper examines this question in case the in-

Communicated by G. Leitmann.

We would like to thank Andrzej Skrzypacz, Hans Schumacher, Jacco Thijssen, and seminar participants at Trinity College Dublin, University of Ulm, the 10th Annual International Conference on Real Options, the Warsaw Economic Meeting 2006, and the 22nd Congress of European Economic Association for comments and suggestions. Financial support from the Flemish Science Foundation (F.W.O.) grant no. G.0374.04 is gratefully acknowledged.

M. Trojanowska $\cdot$ P.M. Kort $(\bowtie)$

Department of Economics, University of Antwerp, Antwerp, Belgium e-mail: Kort@uvt.nl

P.M. Kort

Department of Econometrics \& Operations Research and CentER, Tilburg University, Tilburg, The Netherlands 
stantaneous rate of return on the project may vary due to, e.g., the presence of technological progress. Motivated by statistical evidence, we assume that, based on the current state of the market, the firm is somehow able to estimate the rate of technological progress, but at the same time the firm suspects that this estimation may turn out to be incorrect in future. For instance, such a situation occurs in today's practice in fast developing industries due to the presence of high frequency shocks linked with forthcoming technological innovations (see [1]).

The model presented in this paper is related to three streams of literature. First, the problem we focus on comes from the standard real options literature (see, e.g., [2] and [3]). The aim is to determine the optimal timing to undertake an irreversible investment in the presence of risk. As usual, we start out from solving the problem under the assumption that the firm has complete confidence in the dynamics of the state variable (profit flow). To reflect implications of technological progress, our analysis focuses on projects with finite life-times. Later, we depart from the standard framework. We weaken the assumption on having perfect confidence about dynamics driving future profit flows from the project. To deal with this question our model incorporates the distinction between risk and uncertainty originating from [4]. Risk is referred to as a situation where the probabilistic model is unique. Knightian uncertainty (called ambiguity in the literature) captures the situation where uncertainty is not reducible to a single probability measure and needs to be characterized by a spectrum of probability measures. Having faced the lack of certainty about the true model, the firm's investment decision is based on priors with respect to the future form of uncertainty. Following [5] we adopt the concept of ambiguity so that the firm's priors are represented by a set of probability measures identifying the formal representation of a diffusion process describing the profit flow. To capture the firm's fear of having to deal with a possibly incorrect representation for the future technological progress we impose that the firm is ambiguity averse.

This approach constitutes an alternative to two commonly used decision frameworks: rational expectations and subjective valuation. As argued in [6], the naive application of the rational expectations equilibrium concept on stochastic growth rate models endows the investor with too much knowledge about future growth. On the other hand, subjective valuation of the investment opportunities would result in the creation of subjective illusions about the form of uncertainty (cf. [7]). For the problem under question, ambiguity aversion offers an advantage over the Bayesian approach because it does not require knowledge of the probability distributions capturing the decision maker's beliefs or Bayesian prior with respect to the unknown shocks linked with forthcoming innovations. The investment decision criterion in our model is derived as if the state variable were governed by the worst-case probability measure among the measures considered, which leads to a maximin decision framework.

We derive the optimal investment trigger for the investment problem employing the dynamic programming approach. We show that ambiguity aversion leads to an erosion of the value of the investment opportunity. Consequently, the value of the option to invest is lower, which reduces the opportunity cost of undertaking the investment. However, this does not necessarily bring about investment acceleration. This distinguishes our findings from [8], who established only the option value reduction result for the case of a perpetual project and showed in this way that the impact of 
ambiguity on optimal investment behavior is drastically different from the impact of risk (volatility). Our result that ambiguity may speed up or delay investment links our paper to [9]. Their contribution builds on a comparison of the effects of different degrees of ambiguity before and after the time of the investment. If uncertainty is fully resolved after the investment is undertaken, then an increase in ambiguity accelerates investment. However, if then ambiguity is still present, the firm may possibly delay the investment.

Our approach assumes that undertaking the investment does not change the level of ambiguity and for the reason of analytical tractability it is assumed that before taking a decision the firm knows what the worst case would be. Still, there are two contradictory effects that govern the investment timing. First, there is the option value effect. It captures the erosion of the value of the investment opportunity due to the presence of Knightian uncertainty, which reduces the investment trigger. Second, there is the present-value effect. This effect raises the trigger, because the higher the degree of ambiguity the lower is the net present value of the project under the worst case scenario. It turns out that combining these effects implies nonmonotonicity of the investment trigger in the level of ambiguity. We further examine the probability that the investment takes place before some prespecified point of time. We find that the reduced value of waiting makes that in the short-run Knightian uncertainty may be investment enhancing. The opposite effect holds in the long-run: the ambiguity averse firm totally refrains from investment with a larger probability than a firm facing no ambiguity.

The problem we solve in this paper is also connected with the literature on discount anomalies appearing in the context of a preference based approach. In order to find the endogenous discount factor implied by preferences, we exploit the close link between recursive multiple-priors and robust control. It has been noted that Bellman equations arising in robust control settings are of the same form as Bellman equations arising from stochastic differential utility maximization (cf. [10] and [11]). We employ this analogy together with a dual representation of the value of a claim under model misspecification known from the robust control approach to asset pricing theory ([12], Chap. 12).

We explicitly derive the stochastic discount factor that results from optimizing investment behavior of the ambiguity averse firm facing a menu of representations for uncertainty and we evaluate a decision criterion a posteriori. While carrying out the ex post analysis we define the endogenous price of risk: a measure of the extra return that the firm demands to bear risk. This measure is considered fair by the uncertainty averse firm: if the worst case occurs then the prices of risk are identical, and consequently the risk is overpriced in all other cases.

The remainder of the paper is organized as follows. Section 2 describes two cases. First, it presents a standard investment problem, where the project's value follows a geometric Brownian motion process and the project has finite life-time. The corresponding optimal investment decision criterion is derived. Second, it develops a model where the firm is uncertain about the correct process describing future revenues, and a robust investment decision rule is derived. Section 3 examines the sensitivity of the main results with respect to the key parameters and presents probability calculations. Section 4 derives the endogenous discount factor for the problem and 
discusses the endogenous price of risk. The last section concludes. The mathematical proofs are gathered in the Appendix.

\section{The Model}

\subsection{The Project with Known Dynamics of the State Variable}

Let time be continuous and indexed by $t \geq 0$. Uncertainty is represented by a filtered probability space $\left(\Omega,\left(\mathcal{F}_{t}\right)_{t \geq 0}, \mathcal{Q}\right)$. The firm faces an investment opportunity yielding a stochastic profit. Profit is modelled as a geometric Brownian motion and its dynamics under $\mathcal{Q}$ is given by

$$
d \pi_{t}=\pi_{t}\left(\mu d t+\sigma d B_{t}\right)
$$

with $\pi_{0}>0$ and where $\mu$ and $\sigma$ are positive constants denoting drift and instantaneous standard deviation, respectively, while $d B_{t}$ is the increment of a Wiener process.

In what follows, we consider the project to have a finite life, $\tau$, rather than that it goes on forever. As argued in [13] and [14], this seems to be more realistic in today's knowledge economy with its fast-changing technology environment. Our analysis however applies to a wider class of problems, including maintenance studies as well. For this reason, we define the discount factor more generally in the sense that it consists of two components: the rate of depreciation $\gamma$ and the interest rate $\rho$. As main sources of depreciation we point out physical decay and technological obsolescence. In other words, the project depreciates through age or use (maintenance problems) or advance of competing technologies (cf. [2], Chap. 6). The discount factor is imposed exogenously and follows a deterministic process

$$
d R_{t}=-(\rho+\gamma) R_{t} d t:=-r R_{t} d t,
$$

where $\mu<r$ in order to assure that the firm wants to invest in the project. The mathematical representation of the market is thus as follows $\left\{\left(R_{t}, \pi_{t}\right),\left(\Omega,\left(\mathcal{F}_{t}\right)_{t \geq 0}, \mathcal{Q}\right)\right\}$.

The firm wants to determine the optimal time to pay a sunk cost $I$ to get access to the profit stream described under (1). ${ }^{1}$ Suppose that the investment be undertaken at time $t$. The value of the project installed, $W_{t}$, equals the expected present value of the profit flow generated by the project during its life-time $\tau$ :

$$
W\left(\pi_{t}\right)=\mathbb{E}^{\mathcal{Q}}\left[\int_{t}^{t+\tau} \frac{R_{s}}{R_{t}} \pi_{s} d s \mid \mathcal{F}_{t}\right]=\frac{\pi_{t}\left(1-e^{-(r-\mu) \tau}\right)}{r-\mu}=: W_{t} .
$$

Applying Itô's lemma to $W\left(\pi_{t}\right)$ implies that the dynamics of the value of the project under $\mathcal{Q}$ satisfies

$$
d W_{t}=W_{t}\left(\mu d t+\sigma d B_{t}\right) \quad \text { with } W_{0}=\pi_{0} \frac{1-e^{-(r-\mu) \tau}}{r-\mu} .
$$

\footnotetext{
${ }^{1} \mathrm{We}$ assume that the project has no salvage value, i.e. $I$ is totally sunk. As claimed in [15], second hand markets are of great importance for a nongrowing steady state economy. The fast-changing technology environment constitutes the complementary case where the second hand value is negligible.
} 
The value of the opportunity to invest at time $t$ equals

$$
F\left(\pi_{t}\right)=\max _{t^{\prime} \geq t} \mathbb{E}^{\mathcal{Q}}\left[\frac{R_{t^{\prime}}}{R_{t}}\left(\int_{t^{\prime}}^{t^{\prime}+\tau} \frac{R_{s}}{R_{t^{\prime}}} \pi_{s} d s-I\right) \mid \mathcal{F}_{t}\right] .
$$

Because the model is stationary (see Appendix 6.1) the solution follows by employing standard methods (cf. [2]). The main result of this subsection is summarized in the proposition below.

Proposition 2.1 Let $r>\mu$ and let $\beta$ denote the positive root of the fundamental quadratic $\frac{1}{2} \sigma^{2} \beta(\beta-1)+\mu \beta-r$. The firm optimally undertakes the investment the first time the process $W_{t}$ exceeds the threshold $W^{*}=\frac{\beta}{\beta-1} I$. The corresponding value function equals

$$
F\left(W_{t}\right)= \begin{cases}\left(W^{*}-I\right)\left(\frac{W_{t}}{W^{*}}\right)^{\beta} & \text { if } W_{t} \leq W^{*} \\ W_{t}-I & \text { if } W_{t}>W^{*}\end{cases}
$$

The accompanying trigger on the process of profit flows equals $\pi^{*}=\frac{\beta}{\beta-1} \times$ $I \frac{r-\mu}{1-\exp (-(r-\mu) \tau)}$.

\subsection{The Investment Policy under Ambiguity Aversion}

In this subsection we generalize the problem from the previous subsection. We assume that the investor somehow constructed a model for technological progress, but suspects that model to be misspecified in the long-run. In order to incorporate the firm's fear about model misspecification, we relax the assumption that the firm have perfect confidence about the process governing the stochastic state variable (profit). Throughout this subsection the profit flow is modelled as the stochastic process from the previous section perturbed with the noise of an unspecified distribution. We assume that the perturbation process does not vanish over time and takes the form of nonzero shocks.

For reasons of mathematical tractability we adopt a noise process that persists in an independently and indistinguishably distributed manner (IID) according to the terminology of [16]. The noise is represented by a process $\theta$ over the range $[-\kappa, \kappa]$. Due to this probabilistic model misspecification, the uncertainty is not reducible to a single probability measure and thus needs to be characterized by a spectrum of probability measures. This situation, namely a framework where the firm is not able to assign a precise probability to future alternatives, is referred to as Knightian uncertainty or ambiguity. Following the seminal work [4], we stress the distinction between uncertainty and risk. The latter relates to the standard real options setting where the dynamics of the shock can be captured by a single probabilistic law (a standard geometric Brownian motion in our paper). The parameter $\kappa$ is crucial for the analysis and describes the ambiguity level or the degree of Knightian uncertainty.

Similar to [5] and [17], we exclude the possibility of learning. This is due to technical reasons: there is no constructive way to accommodate the responsiveness of ambiguity to information arriving continuously over time. Consequently, we impose 
that the firm has acquired all information about market fundamentals at the initial date and it seeks a form of robust decision rule to deal with the unprecise characteristics that may (or may not) appear in the future.

The process $\theta$ serves as a density generator, so ambiguity in continuous time is defined as an expansion of the measure $\mathcal{Q}$ with a set of density generators. Consequently, the uncertainty is represented by a family of filtered probability spaces indexed by the set of density generators $\theta:\left(\Omega,\left(\mathcal{F}_{t}\right)_{t \geq 0}, \mathcal{Q}^{\theta}\right)$. For a formal construction the reader is referred to Appendix 6.2. It follows from the representation of ambiguity and Girsanov's theorem that the stochastic process

$$
d B_{t}^{\theta}=d B_{t}+\theta_{t} d t
$$

is a standard Brownian motion on $\left(\Omega,\left(\mathcal{F}_{t}\right)_{t \geq 0}, \mathcal{Q}^{\theta}\right)$.

This fact allows us to incorporate the noise process affecting the profit flows and state the general representation of the profit flow as follows:

$$
\forall \theta \quad d \pi_{t}=\left(\mu-\sigma \theta_{t}\right) \pi_{t} d t+\sigma \pi_{t} d B_{t}^{\theta} .
$$

This construction restricts the analysis to the set of measures equivalent to a reference probability measure. Consequently, the model uncertainty is transformed into parameter uncertainty after the change of measure. Thus all processes used to describe the profit evolution are equivalent and ambiguity is captured by a spectrum of drift terms. The main limitation of this approach is that Girsanov's theorem applies for finite time intervals only and does not ensure the equivalence of measures over an infinite horizon (cf. [18]). In practice it does not really matter whether the option to invest exists during a sufficiently large time horizon or an infinite one as long as the project has finite life-time. However, imposing a fixed horizon on the existence of the option to invest complicates the analysis due to anomalies appearing close to the maturity date of the option to invest. To avoid these we construct an approximation by imposing an infinite decision horizon. ${ }^{2}$

Returning to the main stream of reasoning, the firm targets a robust decision rule with respect to uncertainty (model misspecification) in order to hedge itself against unfavorable shocks. For this reason, we employ an extremal performance measure (which constitutes a special case of the worst case approach) by adopting the concept of ambiguity aversion (fear of model misspecification). The framework of our paper follows the ambiguity aversion definition by Epstein and coauthors [5, 17], which is recently used by [22] in the context of pricing options. ${ }^{3}$

According to the ambiguity aversion concept plugged into the decision making set-up, the firm's aim is to react optimally to the worst alternative among the possible

\footnotetext{
${ }^{2}$ In the literature alternative approaches exist to deal with this problem. The most common are a numerical valuation of American options assuming fixed and finite horizon (employing a finite differences algorithm), an analytic approximation of the solution to the same problem [19] and the extension of Girsanov's theorem to an uncertain time-horizon proposed in [20]. For the axiomatization of an infinite horizon version of recursive multiple-priors, the reader is referred to [21], Appendix B.

${ }^{3}$ The nature of the problem links our analysis with contributions on robust control and model misspecification $([6,23]$ and $[11])$. The formal discussion on the relationship between these two approaches can be found in [21], Sect. 4. We exploit this link in Sect. 4.
} 
outcomes. By construction (see Appendix 6.2), ambiguity aversion corresponds to the case where the firm assigns the lowest value of the growth rate to the project, such that

$$
d \pi_{t}=(\mu-\kappa \sigma) \pi_{t} d t+\sigma \pi_{t} d B_{t}^{\kappa}
$$

For this reason, in what follows we do not distinguish between the level of Knightian uncertainty and the level of ambiguity aversion. To make the framework mathematically transparent, in this section we define the market as $\left\{\left(R_{t}, \pi_{t}\right),\left(\Omega,\left(\mathcal{F}_{t}\right)_{t \geq 0}, \mathcal{Q}^{\kappa}\right)\right\}$, where $\kappa$ is fixed. It is worth stressing that in the case of a one dimensional set of values for $\theta$, ambiguity aversion restores the market, which is complete. This means that all market dynamics can be described under the single worst case measure and the description of uncertainty is reduced to a filtered probability space $\left(\Omega,\left(\mathcal{F}_{t}\right)_{t \geq 0}, \mathcal{Q}^{\kappa}\right)$. Consequently, ambiguity aversion leads to a transformation of uncertainty to risk. Furthermore, the investor is assumed to be neutral with respect to risk. By employing different criteria towards risk and uncertainty in the sequence of the above described steps, we link to Ellsberg's paradox [24] revealing a difference in responses with respect to risk and uncertainty widely reconciled in behavioral economics.

Now we are ready to outline the main steps of project valuation and the solution to the investment problem under ambiguity. The fact that the firm is ambiguity averse is captured by taking the minimum over all models describing the dynamics of profits (captured by (3)), whereas the attitude towards risk is modelled by taking the expectation with respect to the future realization of the profit flow process. Combining the above with the question of determining the timing to undertake the irreversible investment leads to a 'maxmin' decision framework. ${ }^{4}$ We demonstrate in Appendix 6.2 that the project value under the worst case scenario is described by the following proposition.

Proposition 2.2 Under the worst case scenario, the present value at $t$ of a cash flow stream generated by the project during its life-time $\tau$ equals

$$
W\left(\pi_{t}, t\right)=\min _{\theta \in[-\kappa, \kappa]} \mathbb{E}^{\mathcal{Q}^{\theta}}\left[\int_{t}^{t+\tau} \frac{R_{s}}{R_{t}} \pi_{s} d s \mid \mathcal{F}_{t}\right]=\pi_{t} \frac{1-e^{-(r-\mu+\kappa \sigma) \tau}}{r-\mu+\kappa \sigma},
$$

and its dynamics under the worst case measure, $\mathcal{Q}^{\kappa}$, is given by

$$
d W_{t}=W_{t}(\mu-\kappa \sigma) d t+W_{t} \sigma d B_{t}^{\kappa} \quad \text { with } W_{0}=\pi_{0} \frac{1-e^{-(r-\mu+\kappa \sigma) \tau}}{r-\mu+\kappa \sigma}
$$

\footnotetext{
${ }^{4}$ Let us stress the similarity with robust control theory here: "To attain a robust decision rule, the decision maker modifies the usual Bellman equation by adding another player ('nature') who, by choosing a nearby model to hurt the decision maker, assists the decision maker to find a robust decision rule. Thus, the decision maker devises a robust decision rule by finding a value function that solves a 'maxmin' problem." (cf. [12], Chap. 6).
} 
As shown in Appendix 6.2, the corresponding value of the investment opportunity equals

$$
F\left(W\left(\pi_{t}\right)\right)=\max _{t^{\prime} \geq t} \min _{\theta \in[-\kappa, \kappa]} \mathbb{E}^{\mathcal{Q}^{\theta}}\left[\frac{R_{t^{\prime}}}{R_{t}}\left(\int_{t^{\prime}}^{t^{\prime}+\tau} \frac{R_{s}}{R_{t}} \pi_{s} d s-I\right) \mid \mathcal{F}_{t}\right] .
$$

By employing dynamic programming and Itô's lemma we find that

$$
\frac{1}{2} \sigma^{2} W_{t}^{2} F^{\prime \prime}\left(W_{t}\right)+(\mu-\kappa \sigma) W_{t} F^{\prime}\left(W_{t}\right)-r F\left(W_{t}\right)=0 .
$$

This equation is solved subject to standard boundary conditions (value-matching, smooth-pasting and absorbing barrier condition, respectively):

$$
F\left(W^{*}\right)=W^{*}-I, \quad F^{\prime}\left(W^{*}\right)=1, \quad F(0)=0,
$$

in which $W^{*}$ is the investment trigger. The main result is summarized in the following proposition.

Proposition 2.3 Let the firm be ambiguity averse and let $\kappa>0$ represent the firm's degree of Knightian uncertainty. Let $r>\mu-\kappa \sigma$ and let $\beta$ denote the positive root of the fundamental quadratic $\frac{1}{2} \sigma^{2} \beta(\beta-1)+(\mu-\kappa \sigma) \beta-r$. The firm optimally undertakes the investment the first time the process $W_{t}$ exceeds the threshold $W^{*}=$ $\frac{\beta}{\beta-1} I$. The corresponding value function equals

$$
F\left(W_{t}\right)= \begin{cases}\left(W^{*}-I\right)\left(\frac{W_{t}}{W^{*}}\right)^{\beta} & \text { if } W_{t} \leq W^{*} \\ W_{t}-I & \text { if } W_{t}>W^{*}\end{cases}
$$

In order to provide a complete characterization of the optimal investment rule, we state an analogous proposition in terms of profit flows.

Proposition 2.4 Let the firm be ambiguity averse and let $\kappa>0$ represent the firm's degree of Knightian uncertainty. Let $r>\mu-\kappa \sigma$ and let $\beta$ denote the positive root of the fundamental quadratic $\frac{1}{2} \sigma^{2} \beta(\beta-1)+(\mu-\kappa \sigma) \beta-r$. The firm optimally undertakes the investment the first time the process $\pi_{t}$ exceeds the threshold $\pi^{*}=$ $\frac{\beta}{\beta-1} I \frac{\delta}{1-\exp (-\delta \tau)}$, where $\delta=r-\mu+\kappa \sigma$. The corresponding value function is

$$
G\left(\pi_{t}\right)= \begin{cases}\left(\pi^{*} \frac{1-\exp (-\delta \tau)}{\delta}-I\right)\left(\frac{\pi_{t}}{\pi^{*}}\right)^{\beta} & \text { if } \pi_{t} \leq \pi^{*} \\ \pi_{t} \frac{1-\exp (-\delta \tau)}{\delta}-I & \text { if } \pi_{t}>\pi^{*}\end{cases}
$$

\section{Characterization of Investment Policy A Priori}

The investment policy under ambiguity is determined on the basis of recursive-priors formulated in reply to a possible model misspecification. In this section we examine the impact of ambiguity aversion and finite life-time on the project value and optimal investment timing. As a reference point we consider the model without Knightian uncertainty. 


\subsection{No Ambiguity}

To examine the effect of an increase in risk (volatility) of the project in the absence of Knightian uncertainty we derive the following partial derivatives of the triggers:

$$
\frac{\partial W^{*}}{\partial \sigma}=-\frac{W^{*}}{\beta(\beta-1)} \frac{\partial \beta}{\partial \sigma}>0 \quad \text { and } \quad \frac{\partial \pi^{*}}{\partial \sigma}=\frac{\partial W^{*}}{\partial \sigma} \frac{r-\mu}{1-\exp (-(r-\mu) \tau)}>0 .
$$

Both critical values $W^{*}$ and $\pi^{*}$ increase when $\sigma$ increases. Thus a greater degree of risk $\sigma$ results in an increase of the value of the option to wait. This is a well-known result in the real options literature.

\subsection{Comparative Statics Results under Ambiguity Aversion}

First, we focus on the effect of ambiguity aversion on the time interval before the investment takes place. The threshold level of the present value of the project that triggers investment equals

$$
W^{*}=\frac{\beta}{\beta-1} I, \quad \text { where } \beta=\frac{1}{2}-\frac{\mu-\kappa \sigma}{\sigma^{2}}+\sqrt{\left(\frac{1}{2}-\frac{\mu-\kappa \sigma}{\sigma^{2}}\right)^{2}+\frac{2 r}{\sigma^{2}}}>1 .
$$

For this trigger the comparative statics result is easy to establish. Increasing ambiguity $(\kappa)$ erodes the drift and raises $\beta$. This implies that the hysteresis factor $\frac{\beta}{\beta-1}$ decreases, and so does the investment threshold $W^{*}$. Hence, higher ambiguity implies that, compared to the standard problem from Sect. 2.1, less precise information about the project value becomes available over time, which makes waiting with investment less valuable. Thus higher ambiguity erodes the value of the investment opportunity. This result is in contrast with the impact of risk (volatility) in the standard real options literature. On the other hand, the hysteresis factor $\frac{\beta}{\beta-1}$ remains above 1 , thus invalidation of the simple static NPV rule still holds.

To further investigate the impact of ambiguity on investment, we present comparative statics results for the trigger $\pi^{*}$. This allows us to incorporate the impact of ambiguity on the profit received by the firm after the investment has taken place. The partial derivative of trigger $\pi^{*}$ is

$$
\frac{\partial \pi^{*}}{\partial \kappa}=\underbrace{\frac{\partial \pi^{*}}{\partial \beta} \frac{\partial \beta}{\partial \kappa}}_{\text {option value effect }<0}+\underbrace{\frac{\partial \pi^{*}}{\partial \delta} \frac{\partial \delta}{\partial \kappa}}_{\text {present-value effect }>0}
$$

where $\delta$ is the convenience yield as defined in Proposition 2.4. The above decomposition reveals two effects that govern the comparative statics results. The first term is the option value effect. It describes the impact of the change in the option value due to the change in the degree of Knightian uncertainty. Similarly as for the trigger $W^{*}$, the increase in ambiguity lowers the growth rate of the project. This raises $\beta$ and decreases the factor $\frac{\beta}{\beta-1}$ and thus lowers the investment threshold. The second effect is the present-value effect. This measures the change in the trigger caused by the change in the convenience yield. An increase in the degree of ambiguity lowers 

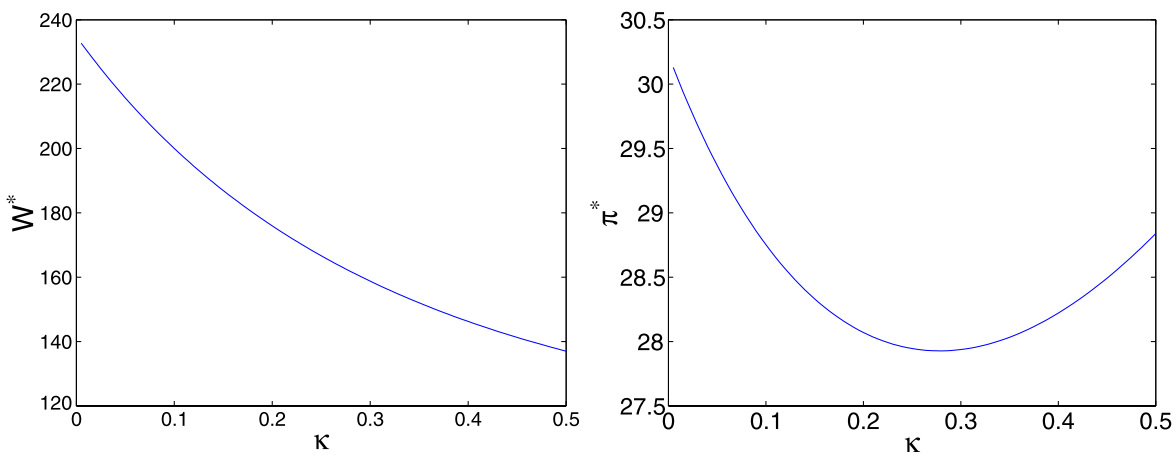

Fig. 1 Impact of ambiguity aversion on triggers $W^{*}$ and $\pi^{*}$. The parameters are $(r, \mu, \sigma$, $\tau, I)=(0.2,0.1,0.2,15,100)$
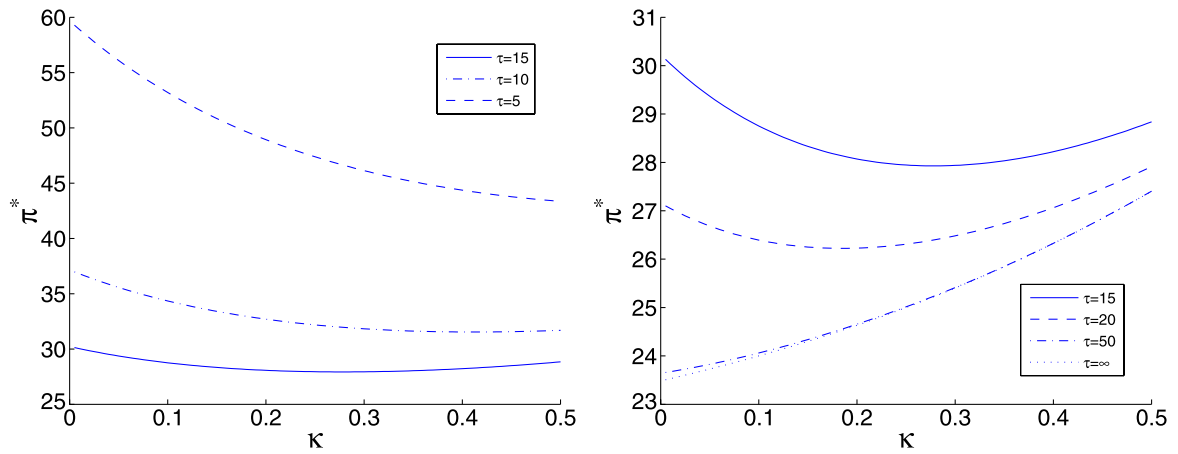

Fig. 2 The finite life-time effect in the optimal investment trigger. The parameters are $(r, \mu, \sigma$, $I)=(0.2,0.1,0.2,100)$

the trend $\mu-\kappa \sigma$ and thus reduces the net present value of the project. This makes investment less profitable and consequently raises the trigger. Thus the present-value effect is contradictory to the option value effect. Combining both effects yields that the relation investment trigger-Knightian uncertainty is equivocal for $\pi^{*}$. It is worth stressing that the origin of the nonmonotonicity result in the investment trigger here is different than in [9]. Their findings are related to the different levels of ambiguity regarding continuation and termination value whereas in our model the level of ambiguity remains unchanged after the investment has been undertaken.

Figure 1 illustrates the difference in the impact of the Knightian uncertainty on the triggers $W^{*}$ and $\pi^{*}$, while this difference will be analyzed further in the next subsection. Nonmonotonicity only occurs in case of $\pi^{*}$ because only then the ambiguity after the investment is taken into account resulting in the present-value effect.

Let us now turn to the effect of the length of the life-time of the project. It is easy to show that the trigger $\pi^{*}$ decreases at an exponential rate with $\tau$. The larger $\tau$, the larger the time that the project generates profits, so that the current profit flow can be smaller for investing to be optimal. Figure 2 depicts the convergence of the optimal threshold to the case of the perpetual one. In the latter case the investment trigger is 
increasing in $\kappa$ so the present-value effect dominates. Moreover, in Fig. 2 we see that the magnitude of the option value effect relative to the present-value effect decays when the life-time of the project increases. For the perpetual project, the comparative statics results for triggers $W^{*}$ and $\pi^{*}$ exhibits monotonicity but in opposite directions: the former is decreasing while the latter is increasing in $\kappa$ (cf. [8]). As said before, this extreme case reflects domination of the present-value effect over the option value effect (see Appendix 6.3). Concluding, we can summarize the comparative statics results for the trigger $\pi^{*}$ in the following proposition.

Proposition 3.1 If the project life-time is finite, the impact of ambiguity on the investment trigger $\pi^{*}$ is equivocal:

$$
\frac{\partial \pi^{*}}{\partial \kappa} \gtreqless 0 \Longleftrightarrow \frac{\frac{\sigma^{2}}{2}(\beta-1)}{\left(\beta-\frac{1}{2}\right) \sigma^{2}+\mu-\kappa \sigma} \gtreqless \frac{\tau \delta}{\exp (\tau \delta)-1} .
$$

Simulations show that a combination of a short life-time of projects and a low degree of Knightian uncertainty is associated with a high value of the trigger $\pi^{*}$. This is also the case when we have high levels of ambiguity and larger life-times.

\subsection{Probability of Investment}

In the previous section we have shown that investment triggers $W^{*}$ and $\pi^{*}$ exhibit different comparative statics results (see Fig. 1), despite the fact that processes $W_{t}$ and $\pi_{t}$ are governed by the same dynamics. To investigate this issue further, we determine the impact of ambiguity aversion on the length of the period before the investment takes place. This allows us to establish how powerful the nonmonotonic effect in $\pi^{*}$ is.

We examine the probability that investment takes within a period of length $H$. Since the future evolution of $\pi$ (and $W$ ) is unknown, the time of the investment is a random variable. Following [25], the cumulative distribution of the first passage time can be written as:

$$
\mathcal{Q}^{\kappa}\left(\sup _{0 \leq t \leq H} W_{t} \geq W^{*}\right)=\Phi\left(d_{1}\right)+\Phi\left(d_{2}\right)\left(\frac{W^{*}}{W_{0}}\right)^{\frac{2 \hat{\mu}}{\sigma^{2}}},
$$

where $d_{1}=\left[-\ln \left(\frac{W^{*}}{W_{0}}\right)+\hat{\mu} H\right][\sigma \sqrt{H}]^{-1}, d_{2}=\left[-\ln \left(\frac{W^{*}}{W_{0}}\right)-\hat{\mu} H\right][\sigma \sqrt{H}]^{-1}, \hat{\mu}=$ $\mu-\kappa \sigma-\frac{\sigma^{2}}{2}$, and $\Phi(\cdot)$ is the standard normal cumulative distribution function. Before we turn to the analysis of the results, let us recall that a change in the degree of ambiguity aversion affects the initial value for the process of the present values of project, $W_{0}$ (see Proposition 2.2), whereas the starting value of the profit flow, $\pi_{0}$, is not sensitive to changes in $\kappa$. While marking both triggers down we obtain $\frac{W^{*}}{W_{0}}=\frac{\pi^{*}}{\pi_{0}}$. Thus, because both $W_{t}$ and $\pi_{t}$ are geometric Brownian motions with identical parameters, the probability of investment is the same for the processes under question. Therefore, without loss of generality, in the remainder of this section we only consider profit flows. 

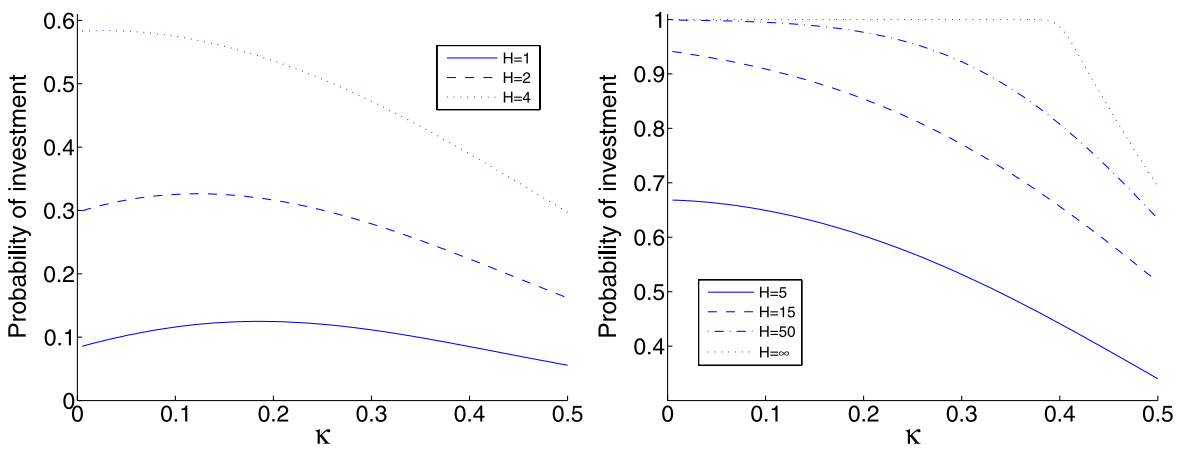

Fig. 3 Impact of ambiguity aversion on probability of investment. The parameters are $(r, \mu$, $\left.\sigma, \tau, I, \pi_{0}\right)=(0.2,0.1,0.2,15,100,20)$

There are two important observations related to the probability of investment. Figure 3 shows the probability of investment for different time horizons and for different levels of ambiguity aversion. The panel for low values of $H$ illustrates the case where the reduced value of waiting makes that in the short-run Knightian uncertainty may be investment enhancing. On the other hand, for relatively high values of $H$ the opposite effect holds: the ambiguous firm is less eager to invest than the firm facing no ambiguity. In fact such a behavior of the probability of investment reflects two opposite effects acting on this measure. First, the investment trigger is decreasing in low values of Knightian uncertainty so that investment occurs earlier. Second, a large degree of Knightian uncertainty lowers the perceived drift of the project. This implies that approaching the zero-absorbing barrier for the process is more likely. Combining these effects imply that, even if the trigger $\pi^{*}$ is nonmonotonic in the degree of the Knightian uncertainty, the probability of investment appears to be monotonically decreasing in the level of Knightian uncertainty for a sufficiently large time horizon.

We focus now on the limiting case as the horizon date $H$ tends to infinity. Following [25], p. 43, we can write:

$$
\mathcal{Q}^{\kappa}\left(\sup _{0 \leq t \leq \infty} \pi_{t} \geq \pi^{*}\right)= \begin{cases}1 & \text { if } \frac{2 \hat{\mu}}{\sigma^{2}} \geq 0 \Leftrightarrow \kappa \leq \frac{\mu-\frac{\sigma^{2}}{2}}{\sigma}, \\ \left(\frac{\pi^{*}}{\pi_{0}}\right)^{\frac{2 \hat{\mu}}{\sigma^{2}}} & \text { if } \frac{2 \hat{\mu}}{\sigma^{2}}<0 \Leftrightarrow \kappa>\frac{\mu-\frac{\sigma^{2}}{2}}{\sigma} .\end{cases}
$$

This confirms the intuitive result that high uncertainty regarding the growth rate of future technological progress lowers the likelihood of the investment, as summarized in the following proposition.

Proposition 3.2 The firm with low degree of ambiguity aversion, $\kappa \leq \frac{\mu-\frac{\sigma^{2}}{2}}{\sigma}$, invests with probability 1 , whereas the firm with large ambiguity aversion, $\kappa>\frac{\mu-\frac{\sigma^{2}}{2}}{\sigma}$, refrains from investment with positive probability, if the horizon date is infinite. 


\subsection{Ironing the Investment Trigger}

Throughout the paper we assume that the life-time of the project is given exogenously and does not reflect any characteristics of market uncertainty. That is, regardless of the form of uncertainty, the project lasts exactly $\tau$ years and then suddenly stops functioning. However, while a firm forms beliefs about the dynamics of profits, at the same time a firm may calculate the estimated life-time of the project reflecting the firm's attitude towards ambiguity.

In this section we provide comparative statics taking this life-time effect into account. We start out by deriving the life-time $\tau(\kappa)$ of the project that guarantees that profits at the moment of investment under the worst case scenario are the same as for the case without ambiguity. The trigger corresponding to this preference-adjusted project's life-time is denoted by $\pi^{*}(\kappa, \tau(\kappa))$, so that $\tau(\kappa)$ is implicitly determined by the equality $\pi^{*}(\kappa, \tau(\kappa)) \equiv \pi^{*}(0, \tau(0))$. The necessary condition for the existence of this iso-profit curve is stated in the proposition below, whereas derivations are placed in Appendix 6.3.

Proposition 3.3 Let $\pi^{*}(\kappa, \infty):=\lim _{\tau \rightarrow \infty} \pi^{*}(\kappa, \tau)$. For levels of ambiguity $\kappa$ satisfying $\pi^{*}(0, \tau(0))>\pi^{*}(\kappa, \infty)$ there exists a $\tau(\kappa)$ such that $\pi^{*}(\kappa, \tau(\kappa)) \equiv$ $\pi^{*}(0, \tau(0))$.

Figure 4 depicts this criterion. If the firm's estimate of the project's life-time is below $\tau(\kappa)$, then the investment trigger on the profit flow process exceeds the investment trigger under the absence of ambiguity. The opposite holds for values of project's life-times above $\tau(\kappa)$. The constraint on the level of ambiguity, $\pi^{*}(0, \tau(0))>\pi^{*}(\kappa, \infty)$, implies that the larger $\tau(0)$ the smaller is the set of $\kappa$ for which the iso-profit curve exists. Consequently, the region where $\pi^{*}(0, \tau(0))>$ $\pi^{*}(\kappa, \tau)$ declines with an increase of the project's life-time $\tau(0)$.

The impact of ambiguity on the investment policy can be analyzed by examining the change in the probability of investment due to a change in the degree of Knightian uncertainty and the adjusted life-time of the project. Simulations for the case where life-time of the project equals $\tau(\kappa)$ suggest that the likelihood of investment is eroded by the presence of Knightian uncertainty. However, establishing in full generality the

Fig. 4 The life-time $\tau(\kappa)$ corresponding with iso-profit curve $\pi^{*}(\kappa, \tau(\kappa))$ $\equiv \pi^{*}(0, \tau(0))$ for the set of parameters

$(r, \mu, \sigma, \tau(0), I)=(0.2,0.1,0.2$, $15,100)$

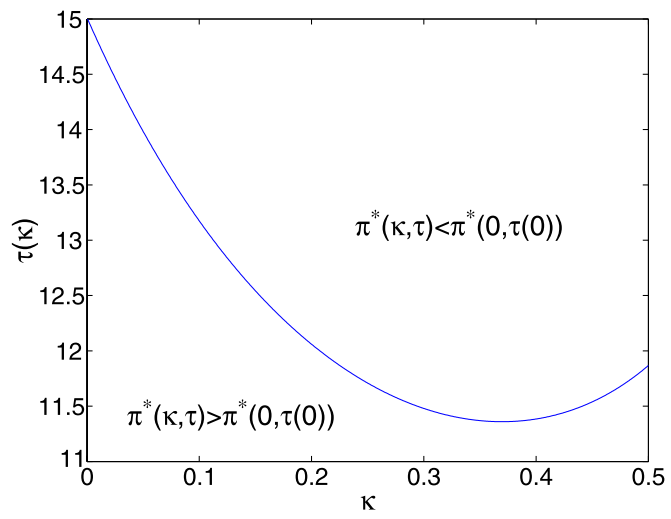


result that the probability of investment over a finite horizon is decreasing in the degree of Knightian uncertainty for projects with life-time $\tau(\kappa)$ remains an open problem (see Appendix 6.3).

\section{Discount Factor Problem and Ex Post Analysis}

\subsection{Endogenous Discount Factor}

It is known from the literature that the application of a preference based valuation may lead to some bias of decision criteria [7]. In this section we examine this problem and we carry out an ex post analysis to validate the investment decision criterion. To do so, we construct the endogenous discount factor implied by the firm's preferences (ambiguity aversion). Because our construction of the preference-adjusted discount factor exploits analogies between ambiguity aversion and the robust control approach to model misspecification, we first discuss the link between recursive-priors and robust control approaches within the framework of our model.

In the absence of misspecification (Sect. 2.1), the optimal investment decision is obtained on the basis of the value of the installed project, $W$, and the accompanying optimal stopping problem. However, under the fear of model misspecification the mathematical representation capturing the value of the installed project is no longer unique (cf. [12], Chap. 12). There are two ways of approaching this problem and they differ in what they take as a model with respect to which conditional expectation is valuated. In Sect. 2.2, we adopt the first approach. We introduce ambiguity in order to model the reaction of the project's growth rate to persistent shocks and we carry out the analysis on the basis of recursive-priors in analogy to [5]. Later, by imposing preferences to reflect the firm's fear of model misspecification (ambiguity aversion) we obtain that the conditional expectation defining the installed project's value, $W$, is evaluated with respect to the worst case model for profit flows. This representation captures a concern about robustness by adjusting the probability distribution of the profit flow.

The second approach to the valuation of the installed project, $W$, is based on an adjustment of the discount factor (cf. [12], Chap. 12). It corresponds to a multiplication of the ordinary discount factor of the no ambiguity case, with the Radon-Nikodym derivative defining the worst case measure. In this way the endogenous discount factor arises from the preferences with respect to ambiguity. ${ }^{5}$

Following [21], from a formal point of view we would like to stress that recursive multiple-priors and a robust optimal control model do not serve as perfect substitutes. The difference stems from alternative restrictions on the initial set of priors and on updating rules. Whereas ambiguity in $[5,16]$ and $[21]$ is captured by a rectangular set of priors updated prior-by-prior (see Appendix 6.2), the robust control model delivers a set of priors constrained by the relative entropy measure (Radon-Nikodym

\footnotetext{
${ }^{5}$ There exists a vast literature on discount factor anomalies in discrete time models (see, e.g. [26] for an up to date summary). The problem of modelling preferences in the real options framework is still at its infancy. Among the pioneering works we have [27-30].
} 
derivative). However, it has been proven (cf. [10] and [11]) that Bellman equations arising from stochastic differential utility optimization and Bellman equations arising in robust control settings are of the same form.

Let us now apply this finding in order to construct the endogenous discount factor for the problem formulated in Sect. 2.2. In order to transform the discount factor used ex ante, $R_{t}$, into the discount factor implied by preferences (called endogenous, ambiguity-adjusted or ex post discount factor in what follows) and denoted by $S_{t}$, we employ a two-step reasoning resembling the search method proposed in [29].

The procedure is designed for a fixed $\kappa$. First, we analyze how a discount factor should be adjusted if only risk is taken into account but no decision making is involved. Technically, we search for a $\mathcal{Q}$-measurable process $S_{t}$, such that it holds that

$$
\mathbb{E}^{\mathcal{Q}}\left[\int_{t}^{t+\tau} \frac{S_{s}}{S_{t}} \pi_{s} d s \mid \mathcal{F}_{t}\right]=\mathbb{E}^{\mathcal{Q}^{\kappa}}\left[\int_{t}^{t+\tau} \frac{R_{s}}{R_{t}} \pi_{s} d s \mid \mathcal{F}_{t}\right], \quad \text { for all } \kappa>0
$$

Restricting to a $\mathcal{Q}$-measurable processes can be interpreted as imposing some stochastic noise on the existing discount factor $R_{t}$. This corresponds with the construction of a Radon-Nikodym derivative suggested by the robust control approach.

Second, we include the decision making aspect. Namely, we verify that the optimal stopping problem solved under $\mathcal{Q}$ with stochastic discount factor $S_{t}$ gives the same decision criterion as the optimal stopping problem solved under $\mathcal{Q}^{\kappa}$ and deterministic discount factor $R_{t}$. The main result is formalized in the proposition below, whereas formal derivations are placed in Appendix 6.4.

Proposition 4.1 There exists an endogenous discount factor satisfying criterion (4) and yielding the same decision criterion under $\mathcal{Q}$ as the worst case approach from Sect. 2.2. This endogenous stochastic discount factor described under $\mathcal{Q}$ follows the geometric Brownian motion

$$
d S_{t}=S_{t}\left(-r d t-\kappa d B_{t}\right), \quad \text { with } S_{0}=0 .
$$

This class of discount factors has been introduced to real options in [30]. Proposition 4.1 establishes a close connection between optimal investment decisions in two frameworks: preference-free valuation under the stochastic discount factor and valuation under ambiguity aversion and the deterministic discount factor.

\subsection{Endogenous Price of Risk}

Having established Proposition 4.1 we proceed to an ex post analysis. To do so, we refer to the concept of the market price of risk known from stochastic finance (see [31], Chap. 15 or [32], Chap. 5). The complete market has the property that the market price of risk is uniquely determined within the model and there is a one-to-one correspondence between the underlying martingale measure and the market price of risk. In the framework of this chapter such a situation occurs for instance if there is no noise related to forthcoming innovations, so that the model without ambigu- 
ity (as in Sect. 2.1) applies. Then, the discounted profit flow follows under $\mathcal{Q}$ the process

$$
d\left(R_{t} \pi_{t}\right)=(\mu-r) R_{t} \pi_{t} d t+\sigma R_{t} \pi_{t} d B_{t}=\sigma R_{t} \pi_{t}\left(\eta d t+d B_{t}\right),
$$

where $\eta=\frac{\mu-r}{\sigma}$ describes the market price of risk.

If the project is subject to shocks that reflect the forthcoming innovations (see Sect. 2.2), so the firm faces ambiguity about the profit on the project, then the market is perceived as incomplete. Market incompleteness is associated with the spectrum of martingale measures that can be adopted in determination of a fair value of the project and consequently results in multiplicity of the market prices of risk. Reference [31] points out that in the framework where the individual preferences of the decision maker are defined and the value functions consistent with these preferences are specified, the market price of risk is defined endogenously. However, the word 'market' appearing in this context in the term 'the market price of risk' is controversial. ${ }^{6}$ Bearing this in mind, in the further discussion we use the term 'the endogenous price of risk' to stress connotation with preferences of the decision maker.

In our framework the description of the individual preferences with respect to ambiguity is essentially equivalent to a specification of the endogenous price of risk, because there is a one-to-one correspondence between the worst case measure describing the ambiguity aversion and the endogenous price of risk. Consequently, the discounted profit flow under $\mathcal{Q}^{\kappa}$ defines the endogenous price of risk $\eta(\kappa)$ as follows:

$$
d\left(R_{t} \pi_{t}\right)=(\mu-\sigma \kappa-r) R_{t} \pi_{t} d t+\sigma R_{t} \pi_{t} d B_{t}^{\kappa}=\sigma R_{t} \pi_{t}\left(\eta(\kappa) d t+d B_{t}^{\kappa}\right) .
$$

Alternatively, we can employ the endogenous discount factor which leads to the same result since under $\mathcal{Q}$ we have:

$$
d\left(S_{t} \pi_{t}\right)=\sigma S_{t} \pi_{t}\left(\eta(\kappa) d t+d B_{t}^{\kappa}\right) .
$$

Let $\delta$ be the convenience yield defined in Proposition 2.4. Clearly, the endogenous price of risk

$$
\eta(\kappa)=\frac{\mu-\kappa \sigma-r}{\sigma}=-\frac{\delta}{\sigma}
$$

is a measure of the extra return, or risk premium, that the firm demands to bear risk.

Suppose we know the true process, $\left(\bar{\theta}_{t}\right)_{t \geq 0}$, capturing the impact of technological progress on the investment. The corresponding profit flow process is:

$$
d \pi_{t}=\left(\mu-\sigma \bar{\theta}_{t}\right) \pi_{t} d t+\sigma \pi_{t} d B_{t}^{\bar{\theta}} .
$$

\footnotetext{
${ }^{6}$ First, the market price of risk is essentially set by the market while here it results from theoretical considerations. Second, the project may not be traded in capital market. "Since the market price of risk is determined by the market, then if there is no market, there is no market price of risk" (cf. [31]). To avoid this paradox in the framework of real options models additional assumptions are imposed to assure the existence of real assets that may (partly) span the risk. For an extensive discussion on the real options models in incomplete markets (with partly spanning assets) the reader is referred to [33] and [34].
} 
Thus, the discounted profit flow process follows:

$$
d\left(R_{t} \pi_{t}\right)=\left(\mu-\sigma \bar{\theta}_{t}-r\right) R_{t} \pi_{t} d t+\sigma R_{t} \pi_{t} d B_{t}^{\bar{\theta}}=\sigma R_{t} \pi_{t}\left(\eta\left(\bar{\theta}_{t}\right) d t+d B_{t}^{\bar{\theta}_{t}}\right),
$$

where $\eta\left(\bar{\theta}_{t}\right)=\frac{\mu-\sigma \bar{\theta}_{t}-r}{\sigma}$ describes the market price of risk. By direct comparison of the risk measures under ambiguity aversion and in the true world we obtain:

$$
\eta(\kappa) \leq \eta\left(\bar{\theta}_{t}\right)
$$

with equality iff $\bar{\theta}_{t} \equiv \kappa$. Intuitively, the worst case approach describes a game against nature that the uncertainty averse firm would consider as fair: if the worst case occurs then the prices of risk are identical, and consequently it is a favorable game in all other cases. ${ }^{7}$ Alternatively, in the language of quantitative finance (see, e.g. [36]) from (6) it follows that $\eta\left(\bar{\theta}_{t}\right)-\eta(\kappa) \geq 0$ describes the difference in the return in excess of the risk-free rate that the ambiguity averse firm gets as compensation for taking risk.

Let $A=\{\kappa: \kappa>0$ and $r>\mu-\kappa \sigma\}$. In particular it holds that

$$
\forall_{\kappa \in A} \quad \eta(\kappa)<\eta,
$$

which describes the relation between prices of risk corresponding to the processes $\left(S_{t} \pi_{t}\right)_{t \geq 0}$ and $\left(R_{t} \pi_{t}\right)_{t \geq 0}$ defined under $\mathcal{Q}$. Thus, (7) says that the uncertainty averse firm perceives the project as more risky since the excess return to the risk ratio determined under ambiguity aversion is lower than in the absence of ambiguity.

\section{Conclusions}

This paper examines the problem of an investment decision under uncertainty in the presence of high frequency shocks linked with, e.g., forthcoming innovations. Because the naive application of the rational expectations equilibrium concept endows the investor with too much knowledge about future growth prospects, we adopt the concept of ambiguity aversion and the concept of model misspecification. This allows us to capture the fear of model misspecification in the decision making process.

Our main focus is on the impact of ambiguity on the investment timing. We identify two factors that critically influence the results: the length of the project's life and whether undertaking the investment solves ambiguity or not. We find that if the investor remains ambiguous about the profit flows after the investment is undertaken, then under a short life-time of the project, ambiguity may be investment enhancing. In general, we obtain that a combination of ambiguity and finite life-time of the project affects the investment trigger equivocally.

\footnotetext{
${ }^{7}$ Because there is one-to-one relation between market price of risk and priors described by density generators (see, Appendix 6.2) similar result can be stated in terms of martingales: "A minimax martingale models a game against the nature that an uncertainty-averse agent would consider as 'fair': in the worst case, it is a fair game (martingale) — as a consequence it must be a favorable game (submartingale) in all other cases" [35].
} 
To investigate the impact of the threshold's nonmonotonicity we examine the probability of investment over a finite horizon. We find that in the short-run ambiguity aversion may be investment enhancing. However, it is also found that the nonmonotonic effect is eroded when the time horizon is increased and in the long-run a high degree of ambiguity aversion results in totally refraining from the investment with a substantial probability.

Due to the fact that our robust decision rule corresponds with a specific form of preferences and may be biased, we evaluate our decision criterion by employing an ex post analysis. We explicitly derive the stochastic discount factor that results from optimizing investment behavior of the ambiguity averse firm facing a menu of representations for uncertainty. The ex post analysis of the investment decision criterion under ambiguity reveals that the uncertainty averse firm perceives the project as more risky than in the absence of ambiguity. In general, the risk is overpriced under ambiguity aversion.

Open Access This article is distributed under the terms of the Creative Commons Attribution Noncommercial License which permits any noncommercial use, distribution, and reproduction in any medium, provided the original author(s) and source are credited.

\section{Appendix}

\subsection{Value of the Project under Risk and Finite Lifetime $\tau$}

Solving (1), we obtain the profit stream:

$$
\pi_{s}=\pi_{t} \exp \left(\int_{t}^{s}\left(\mu-\frac{\sigma^{2}}{2}\right) d u+\int_{t}^{s} \sigma d B_{u}\right),
$$

where $\pi_{0}$ is given and the indices are ordered as $0 \leq t \leq s$. By Fubini's theorem and substitution of $\pi_{t}$ from (8) we get

$$
\begin{aligned}
W\left(t, \pi_{t}\right) & =\mathbb{E}^{\mathcal{Q}}\left[\int_{t}^{t+\tau} e^{-r(s-t)} \pi_{s} d s \mid \mathcal{F}_{t}\right]=\int_{t}^{t+\tau} \mathbb{E}^{\mathcal{Q}}\left[e^{-r(s-t)} \pi_{s} \mid \mathcal{F}_{t}\right] d s \\
& =\pi_{t} \int_{t}^{t+\tau} e^{-r(s-t)} \exp \left(\int_{t}^{s}\left(\mu-\frac{\sigma^{2}}{2}\right) d u\right) \mathbb{E}^{\mathcal{Q}}\left[\exp \left(\int_{t}^{s} \sigma d B_{u}\right) \mid \mathcal{F}_{t}\right] d s \\
& =\pi_{t} \int_{t}^{t+\tau} \exp \left(\left(\mu-r-\frac{\sigma^{2}}{2}\right)(s-t)\right) \mathbb{E}^{\mathcal{Q}}\left[\exp \left(\sigma\left(B_{s}-B_{t}\right)\right) \mid \mathcal{F}_{t}\right] d s \\
& =\pi_{t} \int_{t}^{t+\tau} \exp \left(\left(\mu-r-\frac{\sigma^{2}}{2}\right)(s-t)\right) \exp \left(\frac{\sigma^{2}(s-t)}{2}\right) d s \\
& =\frac{\pi_{t}\left(1-e^{-(r-\mu) \tau}\right)}{r-\mu} .
\end{aligned}
$$

Thus $W\left(\pi_{t}, t\right)$ is independent of time and follows the process denoted by $W_{t}$ :

$$
W\left(\pi_{t}, t\right)=\mathbb{E}^{\mathcal{Q}}\left[\int_{t}^{t+\tau} e^{-r(s-t)} \pi_{s} d s \mid \mathcal{F}_{t}\right]=\frac{\pi_{t}\left(1-e^{-(r-\mu) \tau}\right)}{r-\mu}:=W_{t} .
$$


The value function is derived by splitting the decision between the immediate investment and waiting for a short time interval $t+d t$ as follows:

$$
\begin{aligned}
F_{t}=\max _{t^{\prime} \geq t} \mathbb{E}^{\mathcal{Q}}\left[\int_{t^{\prime}}^{t^{\prime}+\tau} e^{-r(s-t)} \pi_{s} d s-e^{-r\left(t^{\prime}-t\right)} I \mid \mathcal{F}_{t}\right] \\
=\max \left\{\mathbb{E}^{\mathcal{Q}}\left[\int_{t}^{t+\tau} e^{-r(s-t)} \pi_{s} d s \mid \mathcal{F}_{t}\right]-I,\right. \\
\left.\max _{t^{\prime} \geq t+d t} \mathbb{E}^{\mathcal{Q}}\left[\int_{t^{\prime}}^{t^{\prime}+\tau} e^{-r(s-t)} \pi_{s} d s-e^{-r\left(t^{\prime}-t\right)} I \mid \mathcal{F}_{t}\right]\right\} .
\end{aligned}
$$

Inserting $W_{t}$ from (9) and applying the tower property, give that $F_{t}$ is equal to

$$
\begin{gathered}
\max \left\{W_{t}-I, e^{-r d t} \max _{t^{\prime} \geq t+d t} \mathbb{E}^{\mathcal{Q}}\left[\mathbb { E } ^ { \mathcal { Q } } \left[\int_{t^{\prime}}^{t^{\prime}+\tau} e^{-r(s-t-d t)} \pi_{s} d s\right.\right.\right. \\
\left.\left.\left.-e^{-r\left(t^{\prime}-t-d t\right)} I \mid \mathcal{F}_{t+d t}\right] \mid \mathcal{F}_{t}\right]\right\} \\
\Longleftrightarrow F_{t}=\max \left\{W_{t}-I, e^{-r d t} \mathbb{E}^{\mathcal{Q}}\left[d F_{t} \mid \mathcal{F}_{t}\right]\right\} .
\end{gathered}
$$

By the first order Taylor approximation of $e^{-r d t}$ we obtain

$$
F\left(W_{t}\right)=\max \left\{W_{t}-I, \mathbb{E}^{\mathcal{Q}}\left[d F_{t} \mid \mathcal{F}_{t}\right]+F\left(W_{t}\right)-r F\left(W_{t}\right) d t\right\} .
$$

In the continuation region it holds that $r F\left(W_{t}\right) d t=\mathbb{E}^{\mathcal{Q}}\left[d F_{t} \mid \mathcal{F}_{t}\right]$, which by Itô's lemma simplifies to

$$
\frac{1}{2} \sigma^{2} W_{t}^{2} F^{\prime \prime}\left(W_{t}\right)+\mu W_{t} F^{\prime}\left(W_{t}\right)-r F\left(W_{t}\right)=0 .
$$

Solving the above equation subject to appropriate boundary conditions (valuematching, smooth-pasting, and absorbing-barrier condition, respectively):

$$
F\left(W^{*}\right)=W^{*}-I, \quad F^{\prime}\left(W^{*}\right)=1, \quad F(0)=0
$$

gives the unique solution reported in Proposition 2.1.

\subsection{Ambiguity and Ambiguity Aversion in Continuous Time}

This subsection outlines the concept of Knightian uncertainty in continuous time as proposed in [5]. Some necessary definitions used in further subsections to model multiple-priors are also provided here.

Let $\mathcal{L}$ be the set of real-valued, measurable and $\left(\mathcal{F}_{t}\right)$-adapted stochastic processes on $\left(\Omega, \mathcal{F}_{T}, \mathcal{Q}\right)$ with index set $[0, T]$. Let $\mathcal{L}^{2}$ be a subset of $\mathcal{L}$ :

$$
\mathcal{L}^{2}=\left\{\left(\theta_{t}\right)_{0 \leq t \leq T} \in \mathcal{L} \mid \int_{0}^{T} \theta_{t}^{2} d t<\infty \mathcal{Q} \text {-a.s. }\right\} .
$$


Given $\theta=\left(\theta_{t}\right) \in \mathcal{L}^{2}$, define a stochastic process $\left(z_{t}^{\theta}\right)_{0 \leq t \leq T}$ by

$$
d z_{t}^{\theta}=-z_{t}^{\theta} \theta_{t} d B_{t} \quad \text { with } z_{0}^{\theta}=1 .
$$

A stochastic process $\left(\theta_{t}\right) \in \mathcal{L}^{2}$ is called a density generator if $\left(z_{t}^{\theta}\right)$ is a $\left(\mathcal{F}_{t}\right)$ martingale. The most commonly used sufficient condition for $\left(z_{t}^{\theta}\right)$ to be a $\left(\mathcal{F}_{t}\right)$ martingale is Novikov's condition:

$$
\mathbb{E}^{\mathcal{Q}}\left[\exp \left(\frac{1}{2} \int_{0}^{T} \theta_{s}^{2} d s\right)\right]<\infty .
$$

Let $\theta$ be a density generator. Define $\mathcal{Q}^{\theta}$ by

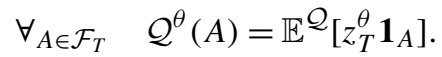

$\mathcal{Q}^{\theta}$ is a probability measure equivalent to $\mathcal{Q}$. Any equivalent to $\mathcal{Q}$ probability measure can be obtained by a density generator as described above.

Let $\Theta$ be a set of density generators. The set of probability measures $\mathcal{P}^{\Theta}$ on $\left(\Omega, \mathcal{F}_{T}\right)$ generated by $\Theta$ is denoted by

$$
\mathcal{P}^{\Theta}=\left\{\mathcal{Q}^{\theta} \mid \theta \in \Theta\right\},
$$

where $\mathcal{Q}^{\theta}$ is derived from $\mathcal{Q}$ according to (10). This completes the formal construction of multiple-priors. Thus, firm's beliefs are operationally captured by the set of probability measures equivalent to $\mathcal{Q}$ in the multiple-priors set-up proposed by [5].

Now, by Girsanov's Theorem, $B_{t}^{\theta}=B_{t}+\int_{0}^{t} \theta_{s} d s$ is Brownian motion relative to $\mathcal{Q}^{\theta}$, and the set of SDEs capturing uncertain profit turns out to be

$$
\begin{aligned}
d \pi_{t} & =\mu \pi_{t} d t+\sigma \pi_{t} d B_{t}=\mu \pi_{t} d t+\sigma \pi_{t}\left(d B_{t}^{\theta}-\theta_{t} d t\right) \\
& =\left(\mu-\sigma \theta_{t}\right) \pi_{t} d t+\sigma \pi_{t} d B_{t}^{\theta}
\end{aligned}
$$

for any $\theta \in \Theta$. Consequently, the multiplicity of measures in $\mathcal{P}$ can be interpreted as modelling ambiguity about the drift of the driving process.

Definition 6.1 A set of density generators is called strongly rectangular if there exists a nonempty compact subset $\mathcal{K}$ of $\mathbb{R}$ and a compact-valued, convex-valued, measurable correspondence $K:[0, T] \rightarrow \mathcal{K}$ such that $0 \in K_{t}$ and

$$
\Theta^{K_{t}}=\left\{\left(\theta_{t}\right) \in \mathcal{L}^{2} \mid \theta_{t}(\omega) \in K_{t} m \otimes \mathcal{Q} \text {-a.s. }\right\}
$$

where $m$ is the Lebesgue measure restricted on $\mathcal{B}([0, T])$.

In the main part of our paper we focus on the case of strongly rectangular sets in which $K_{t}$ is independent of time $t$.

Definition 6.2 The uncertainty characterized by $\Theta^{K}$ is IID uncertainty (see, [16]) if there exists a compact subset $K$ of $\mathbb{R}$ such that $0 \in K_{t}$ and

$$
\Theta^{K}=\left\{\left(\theta_{t}\right) \in \mathcal{L}^{2} \mid \theta_{t}(\omega) \in K m \otimes \mathcal{Q} \text {-a.s. }\right\} .
$$


Definition 6.3 The special case of the IID uncertainty $\Theta^{K}$, where the set $K$ is the symmetric interval $K=[-\kappa, \kappa]$, is called $\kappa$-ignorance.

The real number $\kappa>0$ represents the degree of Knightian uncertainty.

Lemma 6.1 For any $s \geq t$ and for any $\theta \in \Theta^{K_{t}}$ it holds that

$$
\begin{aligned}
\mathbb{E}^{\mathcal{Q}^{\theta}} & {\left[\exp \left(-\int_{t}^{s} \sigma \theta_{u} d u+\sigma\left(B_{s}^{\theta}-B_{t}^{\theta}\right)\right) \mid \mathcal{F}_{t}\right] } \\
\geq \mathbb{E}^{\mathcal{Q}^{\theta^{*}}} & {\left[\exp \left(-\int_{t}^{s} \sigma \theta_{u}^{*} d u+\sigma\left(B_{s}^{\theta^{*}}-B_{t}^{\theta^{*}}\right)\right) \mid \mathcal{F}_{t}\right] }
\end{aligned}
$$

where $\theta_{t}^{*} \equiv \arg \max \left\{\sigma x \mid x \in K_{t}\right\}=\max K_{t}$ is a degenerated measurable process.

For the proof of this lemma the reader is referred to [8], Appendix A. The degeneracy follows from the theory of support functions (cf. [5] or [37], pp. 11-12).

Let $0 \leq t \leq s$ and let $\pi_{0}>0$. Regarding $\mathcal{Q}^{\theta}$ as the true measure, the solution to profit equation (11) admits the following recursive form

$$
\pi_{s}=\pi_{t} \exp \left(\int_{t}^{s}\left(\mu-\frac{\sigma^{2}}{2}\right) d u-\sigma \int_{t}^{s} \theta_{u} d u+\int_{t}^{s} \sigma d B_{u}^{\theta}\right) .
$$

Derivation of the value of the project installed:

$$
\begin{aligned}
W\left(\pi_{t}, t\right)= & \inf _{\theta \in \Theta} \mathbb{E}^{\mathcal{Q}^{\theta}}\left[\int_{t}^{t+\tau} e^{-r(s-t)} \pi_{s} d s \mid \mathcal{F}_{t}\right]=\inf _{\theta \in \Theta} \int_{t}^{t+\tau} \mathbb{E}^{\mathcal{Q}^{\theta}}\left[e^{-r(s-t)} \pi_{s} \mid \mathcal{F}_{t}\right] d s \\
= & \inf _{\theta \in \Theta} \int_{t}^{t+\tau} \pi_{t} e^{\left(\mu-\frac{\sigma^{2}}{2}-r\right)(s-t)} \mathbb{E}^{\mathcal{Q}^{\theta}}\left[\exp \left(\int_{t}^{s} \sigma d B_{u}^{\theta}-\int_{t}^{s} \sigma \theta_{u} d u\right) \mid \mathcal{F}_{t}\right] d s \\
= & \pi_{t} \int_{t}^{t+\tau} e^{\left(\mu-\frac{\sigma^{2}}{2}-r\right)(s-t)} \mathbb{E}^{\mathcal{Q}^{\theta^{*}}}\left[\exp \left(\sigma\left(B_{s}^{\theta^{*}}-B_{t}^{\theta^{*}}\right)-\int_{t}^{s} \sigma \theta_{u}^{*} d u\right) \mid \mathcal{F}_{t}\right] d s \\
= & \pi_{t} \int_{t}^{t+\tau} \exp \left(\left(\mu-r-\frac{\sigma^{2}}{2}\right)(s-t)-\int_{t}^{s} \sigma \theta_{u}^{*} d u\right) \\
& \times \exp \left(\frac{\sigma^{2}(s-t)}{2}\right) d s \\
= & \pi_{t} \int_{t}^{t+\tau} \exp \left((\mu-r)(s-t)-\int_{t}^{s} \sigma \theta_{u}^{*} d u\right) d s .
\end{aligned}
$$

The sequence of equalities is validated by Fubini's theorem, (12), Lemma 6.1, degeneracy of $\theta^{*}$ and easy algebra, respectively.

Lemma 6.2 Let $0 \leq t_{1} \leq t_{2} \leq T$ and let $X$ be an $\mathcal{F}_{T}$-measurable random variable. If $\Theta$ is strongly rectangular then, under the assumption that the minima exist, it holds that

$$
\min _{\theta \in \Theta} \mathbb{E}^{\mathcal{Q}^{\theta}}\left[\mathbb{E}^{\mathcal{Q}^{\theta}}\left[X \mid \mathcal{F}_{t_{2}}\right] \mid \mathcal{F}_{t_{1}}\right]=\min _{\theta \in \Theta} \mathbb{E}^{\mathcal{Q}^{\theta}}\left[\min _{\theta^{\prime} \in \Theta} \mathbb{E}^{\mathcal{Q}^{\theta^{\prime}}}\left[X \mid \mathcal{F}_{t_{2}}\right] \mid \mathcal{F}_{t_{1}}\right]
$$


For the proof of this lemma the reader is referred to [8], Appendix B.

The value function, $F_{t}$, is derived recursively as follows:

$$
\begin{aligned}
F_{t} & =\max _{t^{\prime} \geq t} \min _{\mathcal{Q} \in \mathcal{P}^{\Theta}} \mathbb{E}^{\mathcal{Q}}\left[\int_{t^{\prime}}^{t^{\prime}+\tau} e^{-r(s-t)} \pi_{s} d s-e^{-r\left(t^{\prime}-t\right)} I \mid \mathcal{F}_{t}\right] \\
& =\max \left\{\min _{\mathcal{Q} \in \mathcal{P}^{\Theta}} \mathbb{E}^{\mathcal{Q}}\left[\int_{t}^{t+\tau} e^{-r(s-t)} \pi_{s} d s \mid \mathcal{F}_{t}\right]-I, J_{t}\right\},
\end{aligned}
$$

where

$$
J_{t}=\max _{t^{\prime} \geq t+d t} \min _{\mathcal{Q} \in \mathcal{P}^{\Theta}} \mathbb{E}^{\mathcal{Q}}\left[\int_{t^{\prime}}^{t^{\prime}+\tau} e^{-r(s-t)} \pi_{s} d s-e^{-r\left(t^{\prime}-t\right)} I \mid \mathcal{F}_{t}\right] .
$$

Applying the tower property and Lemma 6.2 yield

$$
\begin{aligned}
J_{t}= & e^{-r d t} \max _{t^{\prime} \geq t+d t} \min _{\mathcal{Q} \in \mathcal{P}^{\Theta}} \mathbb{E}^{\mathcal{Q}}\left[\int_{t^{\prime}}^{t^{\prime}+\tau} e^{-r(s-t-d t)} \pi_{s} d s-e^{-r\left(t^{\prime}-t-d t\right)} I \mid \mathcal{F}_{t}\right] \\
= & e^{-r d t} \max _{t^{\prime} \geq t+d t} \min _{\theta \in \Theta} \mathbb{E}^{\mathcal{Q}^{\theta}}\left[\mathbb { E } ^ { \mathcal { Q } ^ { \theta } } \left[\int_{t^{\prime}}^{t^{\prime}+\tau} e^{-r(s-t-d t)} \pi_{s} d s\right.\right. \\
& \left.\left.-e^{-r\left(t^{\prime}-t-d t\right)} I \mid \mathcal{F}_{t+d t}\right] \mid \mathcal{F}_{t}\right] \\
= & e^{-r d t} \max _{t^{\prime} \geq t+d t} \min _{\theta \in \Theta} \mathbb{E}^{\mathcal{Q}^{\theta}}\left[\operatorname { m i n } _ { \theta ^ { \prime } \in \Theta } \mathbb { E } ^ { \mathcal { Q } ^ { \theta ^ { \prime } } } \left[\int_{t^{\prime}}^{t^{\prime}+\tau} e^{-r(s-t-d t)} \pi_{s} d s\right.\right. \\
& \left.\left.-e^{-r\left(t^{\prime}-t-d t\right)} I \mid \mathcal{F}_{t+d t}\right] \mid \mathcal{F}_{t}\right] \\
= & e^{-r d t} \min _{\theta \in \Theta} \mathbb{E}^{\mathcal{Q}^{\theta}}\left[\operatorname { m a x } _ { t ^ { \prime } \geq t + d t } \operatorname { m i n } _ { \theta ^ { \prime } \in \Theta } \mathbb { E } ^ { \mathcal { Q } ^ { \theta ^ { \prime } } } \left[\int_{t^{\prime}}^{t^{\prime}+\tau} e^{-r(s-t-d t)} \pi_{s} d s\right.\right. \\
& \left.\left.-e^{-r\left(t^{\prime}-t-d t\right)} I \mid \mathcal{F}_{t+d t}\right] \mid \mathcal{F}_{t}\right] \\
= & e^{-r d t} \min _{\theta \in \Theta} \mathbb{E}^{\mathcal{Q}}\left[d F_{t} \mid \mathcal{F}_{t}\right] .
\end{aligned}
$$

Inserting $W_{t}$ and substituting $J_{t}$ give

$$
F_{t}=\max \left\{W_{t}-I, J_{t}\right\}=\max \left\{W_{t}-I, e^{-r d t} \min _{\theta \in \Theta} \mathbb{E}^{\mathcal{Q}}\left[d F_{t+d t} \mid \mathcal{F}_{t}\right]\right\} .
$$

By the first order Taylor approximation of $e^{-r d t}$ we obtain

$$
F\left(W_{t}\right)=\max \left\{W_{t}-I, \min _{\theta \in \Theta} \mathbb{E}^{\mathcal{Q}^{\theta}}\left[d F_{t} \mid \mathcal{F}_{t}\right]+F\left(W_{t}\right)-r F\left(W_{t}\right) d t\right\} .
$$

In the continuation region it holds that

$$
r F\left(W_{t}\right) d t=\min _{\theta \in \Theta} \mathbb{E}^{\mathcal{Q}^{\theta}}\left[d F_{t} \mid \mathcal{F}_{t}\right],
$$


which by Itô's lemma simplifies to

$$
\frac{1}{2} \sigma^{2} W_{t}^{2} F^{\prime \prime}\left(W_{t}\right)+(\mu-\kappa \sigma) W_{t} F^{\prime}\left(W_{t}\right)-r F\left(W_{t}\right)=0 .
$$

\subsection{Comparative Statics Results}

The impact of ambiguity aversion on $\pi^{*}$ :

$$
\frac{\partial \pi^{*}}{\partial \kappa}=\pi^{*}(\underbrace{-\frac{1}{\beta(\beta-1)} \frac{\partial \beta}{\partial \kappa}+\frac{1}{\delta} \frac{\partial \delta}{\partial \kappa}}_{\text {perepetual project effect }>0} \underbrace{-\frac{\tau \exp (-\delta \tau)}{1-\exp (-\delta \tau)} \frac{\partial \delta}{\partial \kappa}}_{\text {finite life-time effect }<0}) .
$$

Note that $\left(\beta-\frac{1}{2}\right) \sigma^{2}+\mu-\kappa \sigma=\sqrt{\left(\mu-\kappa \sigma-\frac{1}{2} \sigma^{2}\right)^{2}+2 r \sigma^{2}}$ and thus

$$
\begin{aligned}
\frac{\partial \beta}{\partial \kappa} & =\frac{1}{\sigma}\left(1-\frac{\mu-\kappa \sigma-\frac{1}{2} \sigma^{2}}{\sqrt{\left((\mu-\kappa \sigma)-\frac{1}{2} \sigma^{2}\right)^{2}+2 r \sigma^{2}}}\right) \\
& =\frac{\sigma \beta}{\sigma^{2}\left(\beta-\frac{1}{2}\right)+\mu-\kappa \sigma}, \\
-\frac{1}{\beta(\beta-1)} \frac{\partial \beta}{\partial \kappa}+\frac{1}{\delta} \frac{\partial \delta}{\partial \kappa} & =-\frac{1}{\beta-1} \frac{\sigma}{\left(\beta-\frac{1}{2}\right) \sigma^{2}+\mu-\kappa \sigma}+\frac{\sigma}{r-\mu+\kappa \sigma} \\
& =\frac{\sigma}{\beta-1} \frac{-r+(\beta-1) \sigma^{2}\left(\beta-\frac{1}{2}\right)+\beta(\mu-\kappa \sigma)}{\left(\left(\beta-\frac{1}{2}\right) \sigma^{2}+\mu-\kappa \sigma\right)(r-\mu+\kappa \sigma)} \\
& =\frac{\sigma}{\beta-1} \frac{\frac{\sigma^{2}}{2}(\beta-1)^{2}}{\left(\left(\beta-\frac{1}{2}\right) \sigma^{2}+\mu-\kappa \sigma\right)(r-\mu+\kappa \sigma)}>0 .
\end{aligned}
$$

The last equality follows after substitution of the fundamental quadratic. Thus, the sign of derivative for $\pi^{*}$ depends on $\tau$ : $\frac{\partial \pi^{*}}{\partial \kappa} \gtreqless 0 \Leftrightarrow \frac{\frac{\sigma^{2}}{2}(\beta-1)}{\left(\beta-\frac{1}{2}\right) \sigma^{2}+\mu-\kappa \sigma} \gtreqless \frac{\tau \delta}{\exp (\tau \delta)-1}$. The RHS can be made large for $\tau$ small, whereas the LHS is independent on $\tau$.

We discuss the condition for ironing the investment trigger:

$$
\frac{\partial \pi^{*}(\kappa, \tau(\kappa))}{\partial \kappa}=\pi^{*}\left(\frac{\partial \ln \left(\frac{\beta(\kappa)}{\beta(\kappa)-1} I \delta(\kappa)\right)}{\partial \kappa}-\frac{\partial \ln (1-\exp (-\delta(\kappa) \tau(\kappa)))}{\partial \kappa}\right) .
$$

Equating the derivative above to 0 and solving the ODE obtained give

$$
\ln \left(\frac{\beta(\kappa)}{\beta(\kappa)-1} I \delta(\kappa)\right)+c_{0}=\ln (1-\exp (-\delta(\kappa) \tau(\kappa))),
$$

where $c_{0}$ is an integration constant. Thus, $C_{0} \frac{\beta(\kappa)}{\beta(\kappa)-1} I \delta(\kappa)=1-\exp (-\delta(\kappa) \tau(\kappa))$ with $C_{0}:=\exp \left(c_{0}\right)$. Rearranging and employing the initial condition reflecting the 
case without ambiguity, yield $C_{0}=\frac{1}{\pi^{*}(0, \tau(0))}$ and

$$
\begin{aligned}
\tau(\kappa) & =\frac{1}{\delta(\kappa)} \ln \left(1-\frac{1}{\pi^{*}(0, \tau(0))} \frac{\beta(\kappa)}{\beta(\kappa)-1} I \delta(\kappa)\right)^{-1} \\
& =\frac{1}{\delta(\kappa)} \ln \left(1-\frac{\pi^{*}(\kappa, \infty)}{\pi^{*}(0, \tau(0))}\right)^{-1} .
\end{aligned}
$$

The solution is meaningful if $\tau(\kappa)>0$, which corresponds to $\pi^{*}(0, \tau(0))>$ $\pi^{*}(\kappa, \infty)$.

We examine the monotonicity (in $\kappa$ ) of the probability of investment over a planing period of length $H$, given that the project's life-time is $\tau(\kappa)$. By definition $\pi^{*}(\kappa, \tau(\kappa))=\pi^{*}(0, \tau(0))$ and thus

$$
\begin{aligned}
& \frac{\partial \mathcal{Q}^{\kappa}\left(\sup _{0 \leq t \leq H} \pi_{t} \geq \pi^{*}(\kappa, \tau(\kappa))\right)}{\partial \kappa} \\
& =\frac{\partial \Phi\left(d_{1}\right)}{\partial d_{1}} \frac{\partial d_{1}}{\partial \kappa}+\frac{\partial \Phi\left(d_{2}\right)}{\partial d_{2}} \frac{\partial d_{2}}{\partial \kappa}\left(\frac{\pi^{*}(0, \tau(0))}{\pi_{0}}\right)^{\frac{2 \hat{\mu}}{\sigma^{2}}} \\
& \quad+\Phi\left(d_{2}\right)\left(\frac{\pi^{*}(0, \tau(0))}{\pi_{0}}\right)^{\frac{2 \hat{\mu}}{\sigma^{2}}} \ln \left(\frac{\pi^{*}(0, \tau(0))}{\pi_{0}}\right) \frac{\partial\left(\frac{2 \hat{\mu}}{\sigma^{2}}\right)}{\partial \kappa} .
\end{aligned}
$$

It is easy to see that the second term is positive, whereas the remaining ones are negative. The total sign of the derivative is difficult to verify analytically.

\subsection{Derivation of Endogenous Discount Factor}

We search for the endogenous discount factor in the class of processes obtained from $R_{t}$ by a stochastic disturbance of the following form:

$$
d S_{t}=d\left(C_{t} R_{t}\right)
$$

where the correction term follows a geometric Brownian motion driven by the same shock as the project under $\mathcal{Q}$, i.e.

$$
d C_{t}=C_{t}\left(\alpha d t-\varkappa d B_{t}\right) .
$$

It follows by Itô's lemma that $S_{t}$ is geometric Brownian motion under $\mathcal{Q}$ :

$$
d S_{t}=S_{t}\left((-r+\alpha) d t-\varkappa d B_{t}\right) .
$$

We impose $S_{0}=1$, which implies that $C_{0}=1$. In order to define a measure, $C_{t}$ has to be a martingale, so it must be the case that $\alpha=0$. Now we are ready to verify condition (4): 


$$
\begin{aligned}
\mathbb{E}^{\mathcal{Q}}\left[\int_{t}^{t+\tau} \frac{S_{s}}{S_{t}} \pi_{s} d s \mid \mathcal{F}_{t}\right] \\
=\pi_{t} \int_{t}^{t+\tau} e^{\left(\mu-\frac{\sigma^{2}}{2}-r-\frac{\varkappa^{2}}{2}\right)(s-t)} \mathbb{E}^{\mathcal{Q}}\left[\exp \left(\int_{t}^{s}(\sigma-\varkappa) d B_{u}\right) \mid \mathcal{F}_{t}\right] d s \\
=\pi_{t} \int_{t}^{t+\tau} e^{\left(\mu-\frac{\sigma^{2}}{2}-r-\frac{\varkappa^{2}}{2}\right)(s-t)} e^{\frac{(\sigma-\varkappa)^{2}}{2}(s-t)} d s \\
=\frac{\pi_{t}\left(1-e^{-(r-\mu+\varkappa \sigma) \tau}\right)}{r-\mu+\varkappa \sigma}=\frac{\pi_{t}\left(1-e^{-\delta \tau}\right)}{\delta}
\end{aligned}
$$

where $\delta=r-\mu+\varkappa \sigma$. Thus, for $\varkappa=\kappa$ we obtain the result from Proposition 2.2.

To verify the second step of the procedure, we extend the solution of the optimal stopping under the stochastic discount factor $S_{t}$ from [30] for the case of a finite life-time $\tau$ of the project. Let

$$
F\left(\pi_{t}\right)=\max _{t^{\prime} \geq t} \mathbb{E} \mathcal{Q}\left[\frac{S_{t^{\prime}}}{S_{t}}\left(\int_{t^{\prime}}^{t^{\prime}+\tau} \frac{S_{s}}{S_{t^{\prime}}} \pi_{s} d s-I\right) \mid \mathcal{F}_{t}\right]
$$

denote the value of investment opportunity at time $t$. Imposing Bellman principle in the form derived by [30], gives that $F(\cdot)$ must satisfy

$$
\mathbb{E}\left[d\left(S_{t} F\left(\pi_{t}\right)\right) \mid \mathcal{F}_{t}\right]=0 \quad \Longleftrightarrow \quad \mathbb{E}\left[F\left(\pi_{t}\right) d S_{t}+S_{t} d F\left(\pi_{t}\right)+d S_{t} d F\left(\pi_{t}\right) \mid \mathcal{F}_{t}\right]=0 .
$$

Consequently, we obtain the following equation

$$
\frac{\sigma^{2}}{2} \pi_{t}^{2} F^{\prime \prime}\left(\pi_{t}\right)+(\mu-\varkappa \sigma) \pi_{t} F^{\prime}\left(\pi_{t}\right)-r F\left(\pi_{t}\right)=0 .
$$

The set of boundary conditions corresponding to the finite life-time $\tau$ is as follows:

$$
F(0)=0, \quad F\left(\pi^{*}\right)=\pi^{*} \frac{1-\exp (-\delta \tau)}{\delta}-I, \quad F_{\pi}\left(\pi^{*}\right)=\frac{1-\exp (-\delta \tau)}{\delta} .
$$

Setting $\varkappa=\kappa$ and solving this standard problem give the same optimal investment criterion as the model under the degree of ambiguity $\kappa$.

\section{References}

1. Cagetti, M., Hansen, L.P., Sargent, T., Williams, N.: Robustness and pricing with uncertain growth. Rev. Financ. Stud. 15, 363-404 (2002)

2. Dixit, A.K., Pindyck, R.S.: Investment under Uncertainty. Princeton University Press, Princeton (1994)

3. McDonald, R., Siegel, D.: The value of waiting to invest. Q. J. Econ. 101, 707-727 (1986)

4. Knight, F.R.: Risk, Uncertainty and Profit. Houghton Mifflin, Boston (1921)

5. Chen, Z., Epstein, L.: Ambiguity, risk and asset returns in continuous time. Econometrica 70, 14031443 (2002)

6. Hansen, L.P., Heaton, J.C., Li, N.: Consumption strikes back? Measuring long-run risk. Mimeo (2005) 
7. Gollier, C.: Optimal illusions and decisions under risk. IDEI Working Papers 340, IDEI, Toulouse (2005)

8. Nishimura, K.G., Ozaki, H.: Irreversible investment and Knightian uncertainty. J. Econ. Theory 136, 668-694 (2007)

9. Miao, J., Wang, N.: Risk, uncertainty and option exercise. Working Paper, Boston University and Columbia University (2006)

10. Skiadas, C.: Robust control and recursive utility. Finance Stoch. 7, 475-489 (2003)

11. Hansen, L.P., Sargent, T.J., Turmuhambetova, G., Williams, N.: Robust control and model misspecification. J. Econ. Theory 128, 45-90 (2006)

12. Hansen, L.P., Sargent, T.J.: Robustness. Princeton University Press, Princeton (2007)

13. Sarkar, S.: The effect of mean reversion on investment under uncertainty. J. Econ. Dyn. Control 28, 377-396 (2003)

14. Gryglewicz, S., Huisman, K.J.M., Kort, P.M.: Finite project life and uncertainty effects on investments. J. Econ. Dyn. Control 32, 2191-2213 (2008)

15. Dobbs, I.M.: Replacement investment: Optimal economic life under uncertainty. J. Bus. Finance Account. 31, 729-757 (2004)

16. Epstein, L., Schneider, M.: IID: Independently and indistinguishable distributed. J. Econ. Theory 113, $32-50(2003)$

17. Epstein, L., Miao, J.: A two-person dynamic equilibrium under ambiguity. J. Econ. Dyn. Control 27, $1253-1288$ (2003)

18. Karatzas, I., Shreve, S.E.: Brownian Motion and Stochastic Calculus. Springer, New York (1991)

19. Barone-Adesi, G., Whaley, R.E.: Efficient analytic approximation of American options values. J. Finance 42, 301-320 (1986)

20. Blanchet-Scalliet, C., El Karoui, N., Martinellini, L.: Dynamic asset pricing theory with uncertain time-horizon. J. Econ. Dyn. Control 29, 1737-1764 (2005)

21. Epstein, L., Schneider, M.: Recursive multiple-priors. J. Econ. Theory 113, 1-31 (2003b)

22. Cont, R.: Model uncertainty and its impact on the pricing of derivative instruments. Math. Finance 16, 519-547 (2006)

23. Anderson, E.W., Hansen, L.P., Sargent, T.J.: A quartet of semigroups for model specification, robustness, prices of risk, and model detection. J. Eur. Econ. Assoc. 1, 68-123 (2003)

24. Ellsberg, D.: Risk, ambiguity and the Savage axioms. Q. J. Econ. 75, 643-669 (1961)

25. Harrison, M.J.: Brownian Motion and Stochastic Flow Systems. Wiley, New York (1985)

26. Fudenberg, D., Levine, D.K.: A dual self model of impulse control. Discussion Paper no. 2112, Harvard University, Cambridge, Massachusetts (2006)

27. Miao, J.: Option exercise with temptation. Econ. Theory 34, 473-501 (2008)

28. Grenadier, S.R., Wang, N.: Investment under uncertainty and time-inconsistent preferences. J. Financ. Econ. 84, 2-39 (2007)

29. Boyarchenko, S., Levendorskii, S.: Discount factors ex post and ex ante, and discounted utility anomalies. Mimeo (2006)

30. Thijssen, J.: Irreversible investment and discounting: An arbitrage pricing approach. Ann. Finance (2009, forthcoming)

31. Björk, T.: Arbitrage Theory in Continuous Time. Oxford University Press, London (2004)

32. Shreve, S.E.: Stochastic Calculus for Finance II. Springer Finance. Springer, Berlin (2004)

33. Henderson, V.: Valuing the option to invest in a incomplete market. Math. Financ. Econ. 1, 103-128 (2007)

34. Thijssen, J.: Incomplete markets, Knightian uncertainty and irreversible investment. Mimeo (2008)

35. Riedel, F.: On optimal stopping under ambiguity. Econometrica (2009, forthcoming)

36. Wilmott, P.: Frequently Asked Questions in Quantitative Finance. Wiley, New York (2006)

37. Asano, T.: Irreversibilities and the optimal timing of environmental policy under Knightian uncertainty. Discussion Paper, Osaka University (2005) 